\title{
Density Functional Study of Electronic, Magnetic and Chemical Bonding Properties of Spinel $\mathrm{CdCr}_{2} \mathrm{O}_{4}$
}

\author{
Bolandhemat $\mathbf{N}^{1 *}$, Md Mahmudur $\mathrm{R}^{1}$, Zainuddin $\mathrm{H}^{\mathbf{2}}$, Chan $\mathrm{KT}^{\mathbf{1}}$ and Shuaibu $\mathrm{A}^{3}$ \\ ${ }^{1}$ Department of Physics, Universiti Putra Malaysia, 43400 UPM Serdang, Selangor, Malaysia \\ ${ }^{2}$ Institutes for Mathematical Research, Universiti Putra Malaysia, 43400 UPM Serdang, Selangor, Malaysia \\ ${ }^{3}$ Department of Physics, Kaduna State University, P. M. B 2309 Kaduna, Nigeria
}

\begin{abstract}
This paper is presented a theoretical study of electronic, magnetic and chemical bonding properties of spinel $\mathrm{CdCr}_{2} \mathrm{O}_{4}$ with a general formula of $\mathrm{AB} 2 \mathrm{X} 4$ ( $\mathrm{A}, \mathrm{B}=$ transition- metal, $\mathrm{X}=$ oxides, chalcogenides) using density functional calculation method combined with spin- polarized theory within generalized gradient approximation. Density functional calculation is performed to observe the effects of magnetic ordering on the electronic and chemical bonding properties of spinel $\mathrm{CdCr}_{2} \mathrm{O}_{4}$ with both cubic and tetragonal structure from a pyrochlore lattice, using Quantum ESPRESSO package. Consequently, in order to investigate the magnetic properties in paramagnetic, ferromagnetic and antiferromagnetic orderings of spinel $\mathrm{CdCr}_{2} \mathrm{O}_{4}$, a first-principles study of the electronic structure as well as chemical bonding properties of spinel $\mathrm{CdCr}_{2} \mathrm{O}_{4}$ compound in two different structural form is performed: the cubic structure in order to investigate the desired properties in paramagnetic and ferromagnetic orderings, and tetragonal structure to calculate the same properties in antiferromagnetic ordering
\end{abstract}

Keywords: Density functional theory; Electronic; Magnetic and chemical bonding properties; Spinels; Spin-polarized theory

\section{Introduction}

Spinel is the magnesium aluminum oxide member of this large group of materials with the following formula, $\mathrm{Mg}^{2+} \mathrm{Al}^{3+} \mathrm{O}^{2-}$. It gives its name to the family of compounds that are identified by two cation sites: an octahedral site and a tetrahedral site [1]. Any material that have the general formula of $\mathrm{A}^{2+}\left[\mathrm{B}^{3+}\right]_{2}\left[\mathrm{X}^{2-}\right]_{4}$ which crystallizes in the face-centered cubic crystal system and are described by the space group $F \overline{d 3} m$ (No. 227) are labeled as a spinel. Spinels are an attracting class of materials that demonstrate rich complex behaviors at ground states. Spineltype compounds with the general formula of $\mathrm{AB}_{2} \mathrm{X}_{4}(\mathrm{~A}, \mathrm{~B}=$ transitionmetal, $\mathrm{X}=$ oxides, chalcogenides) have attracted extensive interest not only due to their diverse properties but also wide applications in electronics, catalysis, magnetism and electrochemical technologies [25]. Spinel oxides $\mathrm{AB}_{2} \mathrm{O}_{4}$ with magnetic $\mathrm{B}$ cations have received special attention due to their identification by three-dimensional geometrical frustration. Spinel oxides with $\mathrm{Cr}^{3+}$ ions on the B sites are good examples to study the frustration. Spinel $\mathrm{CdCr}_{2} \mathrm{O}_{4}$ has been chosen as a model system to study because it has a well-defined magnetic order [6,7]. Spinel $\mathrm{CdCr}_{2} \mathrm{O}_{4}$ is a magnetic compound that forms into a cubic spinel structure, and the magnetic properties stem from the three-dimensional network of $\mathrm{Cr}^{3+}$ magnetic ions, which is indicated as a corner-sharing tetrahedral $[8,9]$. In this paper, density functional calculations are performed to study the effects of magnetic ordering on the electronic and chemical bonding properties of spinel $\mathrm{CdCr}_{2} \mathrm{O}_{4}$ a pyrochlore lattice with magnetic $\mathrm{Cr}$ cations and non-magnetic $\mathrm{Cd}$ cations. The electronic, and chemical bonding properties of spinel $\mathrm{CdCr}_{2} \mathrm{O}_{4}$ with cubic $F \overline{d 3} m$ and tetragonal (I4/amd) structures are calculated using density functional calculation method combined with the spin- polarized theory, and compared the results in both cubic and tetragonal structures for different magnetic orderings. Density functional theory applied with the ground-state theory recovered in the zero temperature limits.

To search for the most stable structure of spinel $\mathrm{CdCr}_{2} \mathrm{O}_{4}$ in term of magnetic ordering, the lowest energy in each structure with different magnetic ordering is calculated. Fallowing that, the effect of magnetism is obtained and analyzed on the basis of total density of states, projected density of states, and charge density distribution within paramagnetic, ferromagnetic and antiferromagnetic orderings using density functional calculations and understanding of the principles of Quantum ESPRESSO in magnetic materials. In continue, to complete the findings of the electronic density of states for spinel $\mathrm{CdCr}_{2} \mathrm{O}_{4}$, the density of states for each atom is calculated, in order to analyze the band gap in each state, separately. Finally, to clarify and compare the chemical bonding properties of spinel $\mathrm{CdCr}_{2} \mathrm{O}_{4}$ in different magnetic orderings, the electronic charge density distribution are obtained for both cubic and tetragonal structures, in the (lll 110$)$ crystallographic planes.

\section{Methodology}

The key starting point in the existing methodology is finding a stable crystal structure of the spinel $\mathrm{CdCr}_{2} \mathrm{O}_{4}$ for both cubic and tetragonal structures as a plane wave basis, followed by obtaining the electronic, magnetic and chemical bonding properties of the compound. Therefore, the structural stability, electronic properties as well as chemical bonding properties of the spinel $\mathrm{CdCr}_{2} \mathrm{O}_{4}$ is calculated within density functional calculation method, using Quantum ESPRESSO package. All pseudopotentials are obtained from the plane wave self-consistent field (PWscf) pseudopotentials online references within generalized gradient approximation parameterized by PerdewBurkew-Enzerhof exchange correlation calculation [10]. Ultrasoft

*Corresponding author: Bolandhemat Najmeh, Department of Physics, Universiti Putra Malaysia, 43400 UPM Serdang, Selangor, Malaysia, Tel: +60143484606; E-mail: bolandhemat.n@gmail.com

Received November 03, 2017; Accepted November 06, 2017; Published November 16, 2017

Citation: Bolandhemat N, Md Mahmudur R, Zainuddin H, Chan KT, Shuaibu A (2017) Density Functional Study of Electronic, Magnetic and Chemical Bonding Properties of Spinel $\mathrm{CdCr}_{2} \mathrm{O}_{4}$. J Material Sci Eng 6: 390. doi: 10.4172/21690022.1000390

Copyright: (C) 2017 Bolandhemat N, et al. This is an open-access article distributed under the terms of the Creative Commons Attribution License, which permits unrestricted use, distribution, and reproduction in any medium, provided the original author and source are credited. 
pseudopotentials are applied for both cubic and tetragonal structures. In the case of generalized gradient approximation, the following valence electrons are considered in this calculation:

- $\quad \mathrm{Cd}^{48}$ (group 12) $1 \mathrm{~s}^{2} 2\left(\mathrm{~s}^{2} \mathrm{p}^{6}\right) 3\left(\mathrm{~s}^{2} \mathrm{p}^{6} \mathrm{~d}^{10}\right) 4\left(\mathrm{~s}^{2} \mathrm{p}^{6} \mathrm{~d}^{10}\right) 5 \mathrm{~s}^{2}($ transition metal): $4 \mathrm{~d}^{10}, 5 \mathrm{~s}^{2}$

- $\mathrm{Cr}^{24}$ (group 6) $1 \mathrm{~s}^{2} 2\left(\mathrm{~s}^{2} \mathrm{p}^{6}\right) 3\left(\mathrm{~s}^{2} \mathrm{p}^{6} \mathrm{~d}^{5}\right) 4 \mathrm{~s}^{1}$ (transition metal): $3 \mathrm{~d}^{5}, 4 \mathrm{~s}^{1}$

- $\quad 1 \mathrm{~s}^{2} 2\left(\mathrm{~s}^{2} \mathrm{p}^{4}\right)$ (nonmetal): $2 \mathrm{~s}^{2}, 2 \mathrm{p}^{4}$.

The crystal structures of spinel $\mathrm{CdCr}_{2} \mathrm{O}_{4}$ is optimized with paramagnetic, ferromagnetic and antiferromagnetic orderings. The crystal structure of spinel $\mathrm{CdCr}_{2} \mathrm{O}_{4}$ is cubic with the space group $F \overline{d 3} m$ (No. 227), and the lattice constant $\left(a=b=c=8.667 \AA, \alpha=\beta=\gamma=90^{\circ}\right)$ in the paramagnetic and ferromagnetic ordering, whereas for the antiferromagnetic ordering is tetragonal with the space group $\mathrm{I}_{1} /$ amd and lattice constant $\left(\mathrm{a}=\mathrm{b}=8.634 \AA\right.$ and $\left.\mathrm{c}=8.694 \AA, \alpha=\beta=\gamma=90^{\circ}\right)$. The calculated data of lattice constant is in a very good match with the experimental results in reference 6 . In this reference, the results is reported $a=8.588$ Å for the cubic phase and $(c-a) / a=0.5 \times 10^{-3}(\mathrm{c}=8.592$ $\AA$ ) for the tetragonal phase from elastic and inelastic neutron scattering measurements on $\mathrm{CdCr}_{2} \mathrm{O}_{4}[6]$.

The lattice parameters of the spinel $\mathrm{CdCr}_{2} \mathrm{O}_{4}$ are demonstrated for both cubic and tetragonal structures, with the $\mathrm{Cr}$ magnetic ions and the Cd non-magnetic and. Crystal Structure is studied using XCrySDen visualization software. The density functional theory together with the general gradient approximation is used to find the electronic ground state properties of spinel $\mathrm{CdCr}_{2} \mathrm{O}_{4}$. It is necessary to know that choosing the suitable crystal structure with suitable symmetry and exact lattice parameters for different magnetic orderings is the first step of the Quantum ESPRESSO calculation, and also by carrying out the self-consistent convergence test, the key of Quantum ESPRESSO calculation is finding the actual energy cut-off and k-point mesh in order to have the better and correct results. In order to calculate the total energy, the exchange and correlation functional is described within the generalized gradient approximation based on exchangecorrelation energy optimization. The optimization of atomic positions and cell parameters is approved through the minimization of energy using Hellman-Feynman forces acting on atoms with the BroydenFlecher-Goldfarb-Shanno scheme and to get the actual relaxed atomic positions and cell parameters for each element the PWscf (VC-relax) input code is applied.

\section{Results}

In order to search for the most stable structure of spinel $\mathrm{CdCr}_{2} \mathrm{O}_{4}$ in the case of magnetic orderings, the total energy in each structure is calculated. The lowest energy in different magnetic orderings can be found from the output file of the scf calculation for three different magnetic ordering. The total energy in paramagnetic, ferromagnetic and antiferromagnetic ordering is -1182.47205850 (Ry), -1182.95939757 (Ry), and -1179.88709055 (Ry), respectively (1 Rydberg $=13.6056981 \mathrm{eV}$ ). From the results of calculation, it can be seen that the Antiferromagnetic ordering of spinel $\mathrm{CdCr}_{2} \mathrm{O}_{4}$ in tetragonal structure is the most stable structure. Taking into account that the total energy in negative and it is just a sign to show that the positive work has to be done on the electron to remove it from that orbit which mean energy will have to be provided to the electron. The negative total energy of the system is just a quantitative sign that shows energy must be added to remove the electron, completely.

To find the charge density distribution of spinel $\mathrm{CdCr}_{2} \mathrm{O}_{4}$ compound, the postprocessing package is used which was initially developed by Paolo Giannozzi, Stefano de Gironcoli, Andrea Dal Corso, and many others [11]. The postprocessing code performs two steps: reads the output file produced by pw.x, extract and calculate the desired quantity, and then writes the desired quantity to file in a suitable format for various types of plotting. Choosing (110) crystallographic plane for both tetragonal structure with lattice parameters $\left(\mathrm{a}=\mathrm{b}=\mathrm{c}, \alpha=\beta=\gamma=90^{\circ}\right)$ and cubic structure with lattice constant $\left(\mathrm{a}=\mathrm{b}=\mathrm{c}, \alpha=\beta=\gamma=90^{\circ}\right)$, is the best choice to compare the charge density distribution as well as chemical bonding properties of both structures in different magnetic orderings. Figure 1 shows the surface of two-dimensional charge density distribution in (110) crystallographic plane for tetragonal structure. The same crystallographic plane has been applied for cubic structure as well. Accordingly, in order to investigate the distribution of the total electronic charge density of $\mathrm{CdCr}_{2} \mathrm{O}_{4}$ compound, the electronic charge density is calculated in (110) crystallographic plane for both cubic and tetragonal structures (Figure 2). From Figure 2, it can be seen that the total electronic charge density in the tetragonal structure is stronger than in the cubic structure by comparing the color of charge density scales in both structures, as the colors stand for charge accumulation and charge deflection between the bonds.

In other words, the difference between minimum and maximum amount of charge density in tetragonal structure is larger than in cubic structure, which is a good proof to admit that the tetragonal structure of spinal $\mathrm{CdCr}_{2} \mathrm{O}_{4}$ is more stable and stronger than the cubic structure, in term of charge density distribution. Also, it can be seen from the color charge density scale that the purple color $(+1.0000)$ corresponds to the maximum charge accumulating site, so the chromium atoms have the greater charge density compare to the other atoms.

Electronic density of states is another important property that gives an appropriate explanation of the effect of magnetism on the electronic properties of spinel $\mathrm{CdCr}_{2} \mathrm{O}_{4}$. And so, in the case of electronic properties, the density of states and projected density of states of spinel
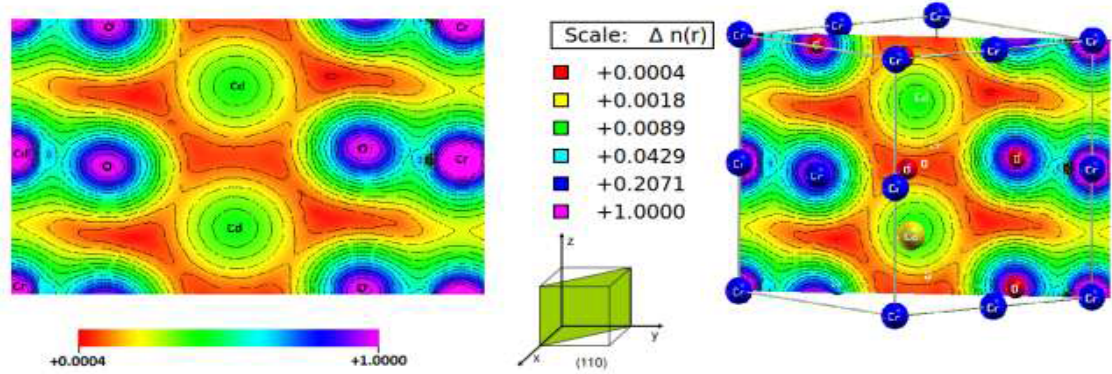

Figure 1: 2D Charge density distribution contour of $\mathrm{CdCr}_{2} \mathrm{O}_{4}$ with tetragonal structure using Xcrysden software. 
(a)

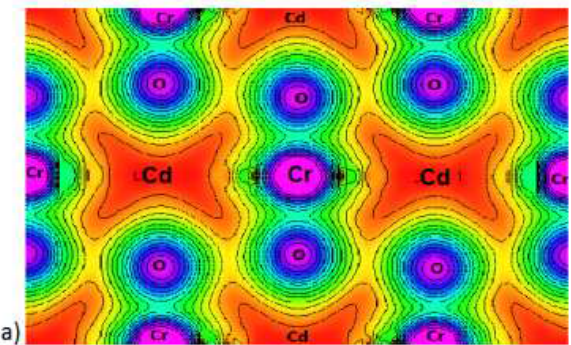

(b)

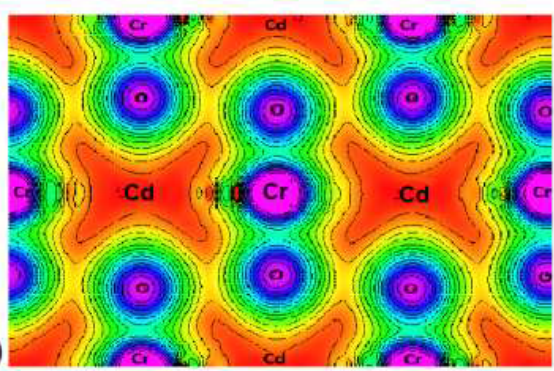

(c)

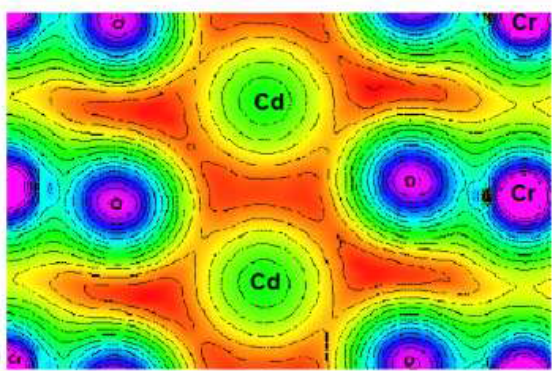

Figure 2: Electronic charge density contour for $\mathrm{CdCr}_{2} \mathrm{O}_{4}$; (a) Paramagnetic ordering, (b) Ferromagnetic ordering, (c) Antiferromagnetic ordering

$\mathrm{CdCr}_{2} \mathrm{O}_{4}$ is calculated, initially without considering the magnetization (Starting-magnetization $=0.0$ ), and then, by considering the parameter of magnetization. The spin-polarized total density of states and projected density of states for spinel $\mathrm{CdCr}_{2} \mathrm{O}_{4}$ is obtained, using $8 \times 8$ $\times 8$ Monkhorst-Pack grid [12]. Figure 3 shows the total density of states of spinel $\mathrm{CdCr}_{2} \mathrm{O}_{4}$ in three different magnetic configurations. By considering the charge density plot and also the total and absolute magnetization values obtained from density of states output files (Total magnetization $=0.0$ and Absolute magnetization=0.0), it can be found that in the paramagnetic configuration, the two spin contributions are exactly the same, as its ground state is non-magnetic. In another word, the valence bands (corresponding to bonding states) are all doubly occupied and the total magnetization is zero. Also, by applying the initial magnetization for $\mathrm{Cr}^{3+}\left(3 \mathrm{~d}^{3}\right)$ magnetic atoms, the same density of states calculation is calculated for ferromagnetic and antiferromagnetic orderings, as well. In the case of ferromagnetic ordering, when the spins are oriented parallel to each other, the magnetic energy is minimized. For that reason, the material may turn into a strong magnet in the presence of an externally applied magnetic field. Also, in the case of antiferromagnetic ordering, the magnetic energy is minimized when pairs of nearby spins are oriented antiparallel to each other. This state proposes that the signs of the magnetic interactions in an antiferromagnet are opposite those in a ferromagnet [13]. From Figure 3, it can be seen that in ferromagnetic ordering (Total magnetization $=12.00$ (Bohr magneton/cell) and Absolute magnetization $=12.45$ (Bohr magneton/cell)), one spin population is larger than the other and the density of states of the two spin are not
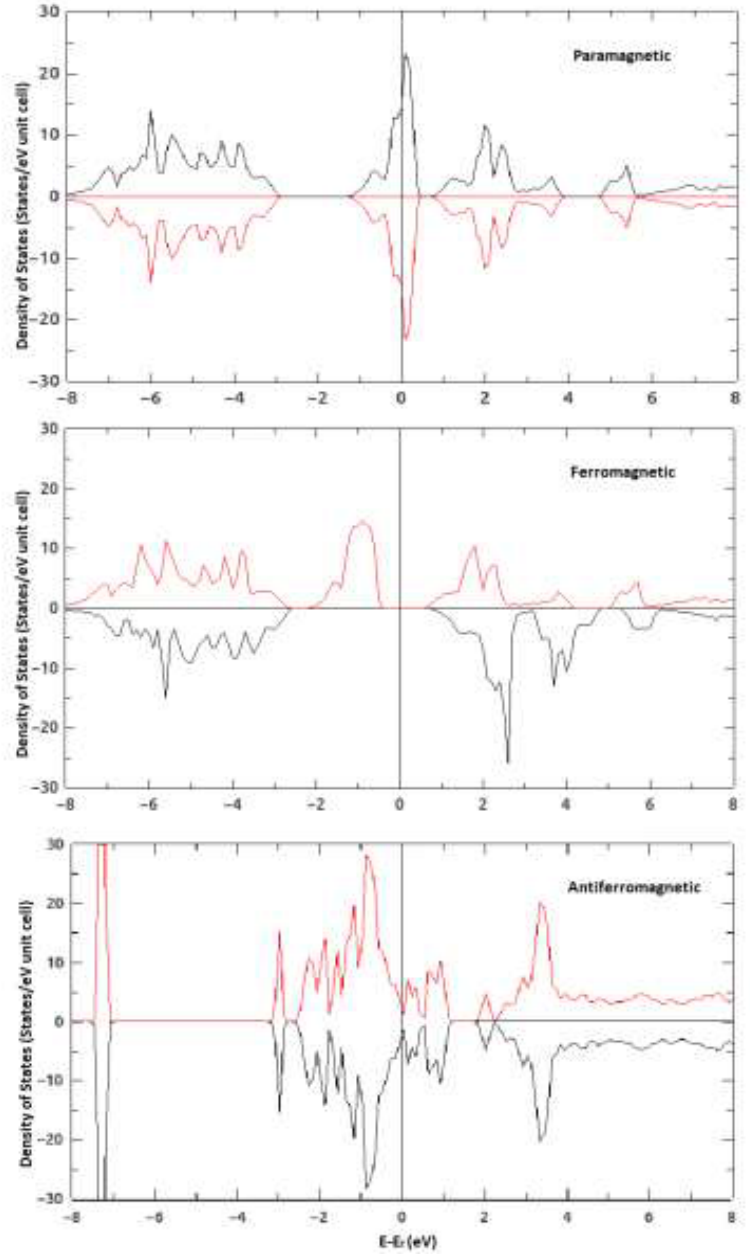

Figure 3: Density of states for $\mathrm{CdCr}_{2} \mathrm{O}_{4}$ in different magnetic configurations. The Fermi level $E_{f}$ is indicated by vertical line.

aligned anymore. Although, in the antiferromagnetic ordering (Total magnetization $=0.0$ and Absolut magnetization $=25.21$ (Bohr magneton/ cell)), half of the atoms have a magnetization that is opposite to the magnetization of the other half, therefore, the total magnetization is zero and the two spin contributions are the same. Also from Figure 3 , it can be found that spinel $\mathrm{CdCr}_{2} \mathrm{O}_{4}$ is metallic in paramagnetic ordering as well as antiferromagnetic ordering, as the density of states can be considered to start at the bottom of the valance band and fill up the Fermi level. Since the conduction band and valance band are overlapped, the Fermi level is in conduction band and there are plenty of electrons available for conducting. While in ferromagnetic ordering, it is semiconductor as in a semiconductor, there is a small gap between the conduction and valence bands and the Fermi level is surrounded by a small band gap $(1 \sim 3 \mathrm{eV})$.

In order to analyze the band gap in each state, the density of states of spinel $\mathrm{CdCr}_{2} \mathrm{O}_{4}$ is calculated for each atom, separately. Figure 4 shows the projected density of states of $\mathrm{CdCr}_{2} \mathrm{O}_{4}$ in paramagnetic ordering. It can be seen from the plot that in Cadmium, from approximately -10 to $-8 \mathrm{eV}, \mathrm{d}$ orbital derived bands, in Chromium, $\mathrm{d}$ orbitals split into two rather sharp peaks in the interval from approximately -2 to $4 \mathrm{eV}$, and in Oxygen, from approximately -21 to $-19 \mathrm{eV}, \mathrm{S}$ orbital derived bands, these bands are somewhat isolated and far away from the other bands 

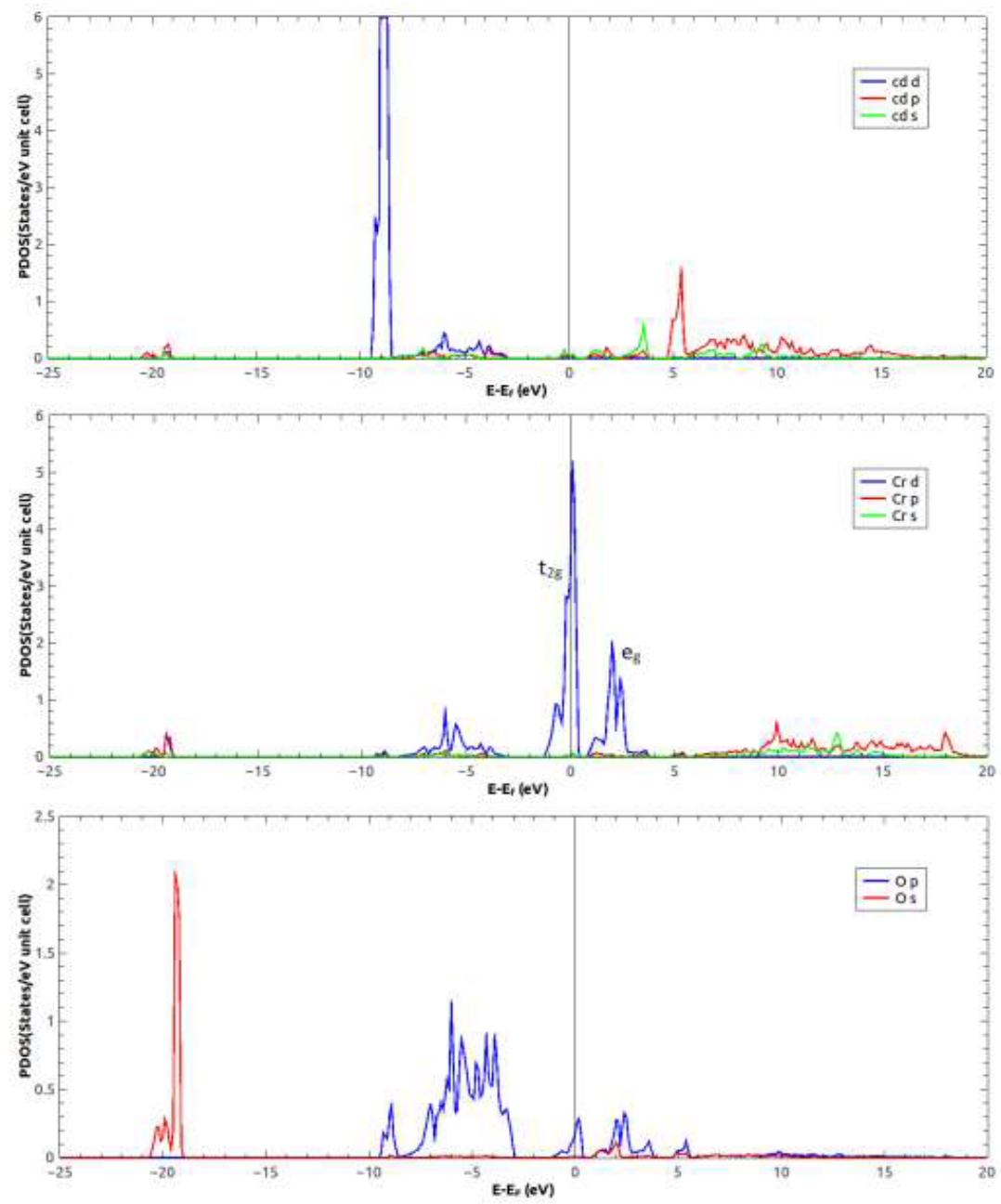

Figure 4: Calculated projected density of states (PDOS) for $\mathrm{CdCr}_{2} \mathrm{O}_{4}$ in paramagnetic ordering.

that we will pay little attention to them, also from approximately -10 to $-3 \mathrm{eV}$, $\mathrm{p}$ orbital derived bands. Therefore, according to the LSDA calculations, the band gap seems to be placed between the two crystal field split $\mathrm{Cr} \mathrm{d}$ manifolds, and the $\mathrm{O} p$ states are deeper. On the other word, by clarifying the band for all orbitals ( $\mathrm{Cd} \mathrm{s} \mathrm{p} \mathrm{d),} \mathrm{(Cr} \mathrm{s} \mathrm{p} \mathrm{d)} \mathrm{and}$ $(\mathrm{O} s \mathrm{P})$, it is clear that the contribution of $\mathrm{s}$ and $\mathrm{p}$ orbitals in charge density for both $\mathrm{Cd}$ and $\mathrm{Cr}$ atoms, can be neglected compare to the contribution of orbital $\mathrm{d}$. In oxygen atom orbital s can be neglected because these bands are isolated and far away from the other bands.

In continue, to observe the effect of magnetization in projected density of states, the projected density of states is plotted for three different magnetic orderings of spinel $\mathrm{CdCr}_{2} \mathrm{O}_{4}$, considering the densities of $\mathrm{Cd} 4 \mathrm{~d}, \mathrm{Cr} 3 \mathrm{~d}$, and $\mathrm{O} 2 \mathrm{p}$ states with the occupied bands in the energy range down to $-10 \mathrm{eV}$ below the Fermi level (Figure 5). Figure 5 shows that the density of states of $\mathrm{Cd}$ and $\mathrm{O}$ atoms in cubic structure of $\mathrm{CdCr}_{2} \mathrm{O}_{4}$ are remaining the same for both paramagnetic and ferromagnetic orderings and only the density of $\mathrm{Cr} \mathrm{d}$ states is changing from paramagnetic to ferromagnetic. However, the density of states of the three atoms is changed in tetragonal structure of $\mathrm{CdCr}_{2} \mathrm{O}_{4}$, but the difference in densities is manly for $\mathrm{Cr}$ atoms. Figure 6 shows the projected density of states of $\mathrm{CdCr}_{2} \mathrm{O}_{4}$ for both spin up and down in ferromagnetic ordering. It can be seen that the charge density in cadmium and oxygen remains the same and only in chromium atom, the population of spin up and spin down is changed as the initial magnetization is applied only on chromium atom. Figure 7 shows the projected density of states calculated for paramagnetic spinel $\mathrm{CdCr}_{2} \mathrm{O}_{4}$. As it is obvious from Figure 7, Cd d states in $\mathrm{CdCr}_{2} \mathrm{O}_{4}$ form a narrow group of bands below the bottom of $\mathrm{O} p$ states, which results in the appearance of a sharp peak of d DOS between -8.5 and $-9.5 \mathrm{eV}$. Therefore, $\mathrm{Cd} d$ states have no magnetic effects, as they are completely occupied and lie well below $\mathrm{E}_{\mathrm{f}} \mathrm{O}$ p-derived bands in $\mathrm{CdCr}_{2} \mathrm{O}_{4}$ spread over the energy range from -8 to $-3 \mathrm{eV}$ and are separated by a gap of $\sim 1.5 \mathrm{eV}$ from $\mathrm{Cr} 3 \mathrm{~d}$ states, which give prevailing contribution to the bands crossing the Fermi level and a prominent DOS peak at $\mathrm{E}_{\mathrm{f}} \mathrm{Cr} \mathrm{d}$ states also are split into a triplet $\mathrm{t}_{2 \mathrm{~g}}$ and a doublet $\mathrm{e}_{\mathrm{g}}$ with two sharp peaks in the range from -2 to $4 \mathrm{eV}$. As it is mentioned earlier, in the transition metal oxides, the $\mathrm{d}$ level is fivefold degenerate. The degeneracy of $\mathrm{d}$ level is split into the lower energy $t_{2 g}$ level and higher energy $e_{g}$ level by the crystal field splitting in an octahedral field which in this system chromium atom octahedrally coordinated by oxygen.

In order to explain the chemical bonding properties of spinel $\mathrm{CdCr}_{2} \mathrm{O}_{4}$, the electronic charge density is calculated in different 
Citation: Bolandhemat N, Md Mahmudur R, Zainuddin H, Chan KT, Shuaibu A (2017) Density Functional Study of Electronic, Magnetic and Chemical Bonding Properties of Spinel $\mathrm{CdCr}_{2} \mathrm{O}_{4}$. J Material Sci Eng 6: 390. doi: 10.4172/2169-0022.1000390
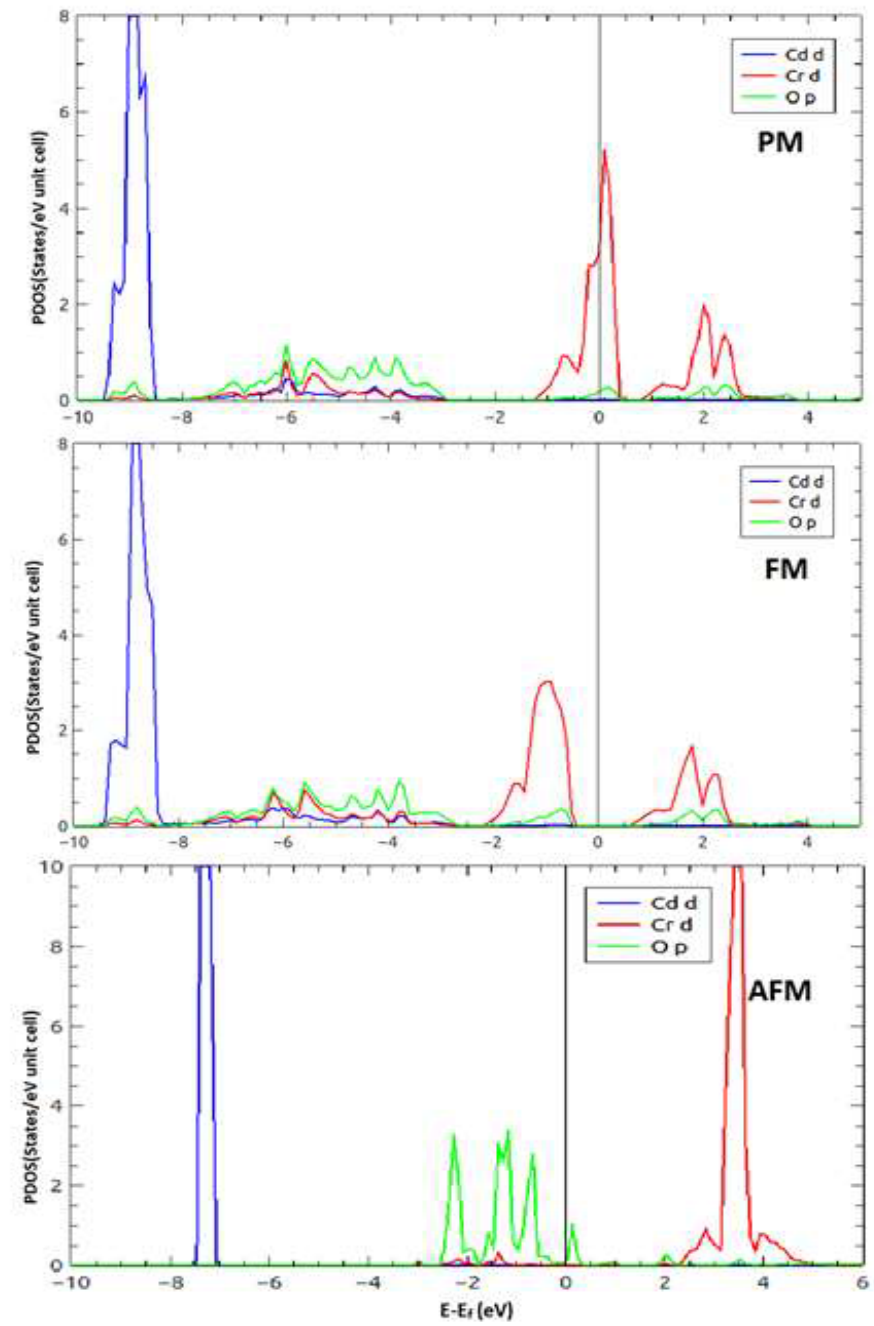

Figure 5: Projected density of states for cubic and tetragonal structure of $\mathrm{CdCr}_{2} \mathrm{O}_{4}$ in different magnetic configurations. The Fermi level $\mathrm{E}_{\mathrm{f}}$ is indicated by vertical line.

magnetic orderings. Figure 8 shows the electronic charge density maps along the (110) crystallographic plane. The bonding has a significant covalent character due to sharing of charge between $\mathrm{O}$ atoms. Moreover, the charge transfer occurs mainly from $\mathrm{Cr}$ atoms towards $\mathrm{O}$ atom. It can be found from Figure 8 that in cubic structure of $\mathrm{CdCr}_{2} \mathrm{O}_{4}$, there is no significant change in electronic charge density contour in both paramagnetic and ferromagnetic orderings, while there is a major transformation in charge density distribution as shifting from cubic to tetragonal structure. Also, in paramagnetic and ferromagnetic orderings of $\mathrm{CdCr}_{2} \mathrm{O}_{4}, \mathrm{Cd}$ and $\mathrm{O}$ atoms shows the ionic nature though the charge density contours around the cadmium is not completely circular but it shows the ionic bonding. Ionic bonding occurs between atoms that have opposite needs for electrons and results in a transfer of electrons. However, covalent bonding occurs between atoms that have similar needs for electrons and results in a sharing of electrons, so, due to sharing of charge between $\mathrm{Cr}$ and $\mathrm{O}$ atoms, $\mathrm{Cr}$-O makes the covalent bonding. Finally, in the cubic structure of $\mathrm{CdCr}_{2} \mathrm{O}_{4}, \mathrm{Cd}$ atom shows a very week charge density but there is the stronger charge density as moving from cubic to tetragonal structure, and also the $\mathrm{Cd}-\mathrm{O}$ makes partial covalent bonding in tetragonal structure. It is another reason to accept that tetragonal structure is the most stable and satisfied structure of spinel $\mathrm{CdCr}_{2} \mathrm{O}_{4}$, knowing that most of the transition metal oxides have strong covalent characteristics.

\section{Conclusion}

In the present work, a computational study of the electronic, magnetic and chemical bonding properties of spinel $\mathrm{CdCr}_{2} \mathrm{O}_{4}$ is reported using density functional theory (DFT) based on first principle performance within Quantum ESPRESSO method, with GGA exchange correlation functional. All the calculations perform in the basis of DFT by applying a plane wave basis set joined with spin-polarized theory. This calculation was able to investigate and observe the electronic and magnetic characteristics in spinel $\mathrm{CdCr}_{2} \mathrm{O}_{4}$. Furthermore, since chromium spinel belongs to transition metals with partially filled d-subshell, for electronic structure calculations, spin polarization had to be taken into account. In addition, the first-principles calculations are used to examine the effects of magnetic orderings on the ground state properties of this compound. It has been found from the outcomes that the most stable structure is the antiferromagnetic spinel $\mathrm{CdCr}_{2} \mathrm{O}_{4}$ with tetragonal structure compares to the other magnetic configurations in terms of energy and charge density distribution, and 
Citation: Bolandhemat N, Md Mahmudur R, Zainuddin H, Chan KT, Shuaibu A (2017) Density Functional Study of Electronic, Magnetic and Chemical Bonding Properties of Spinel $\mathrm{CdCr}_{2} \mathrm{O}_{4}$. J Material Sci Eng 6: 390. doi: 10.4172/2169-0022.1000390

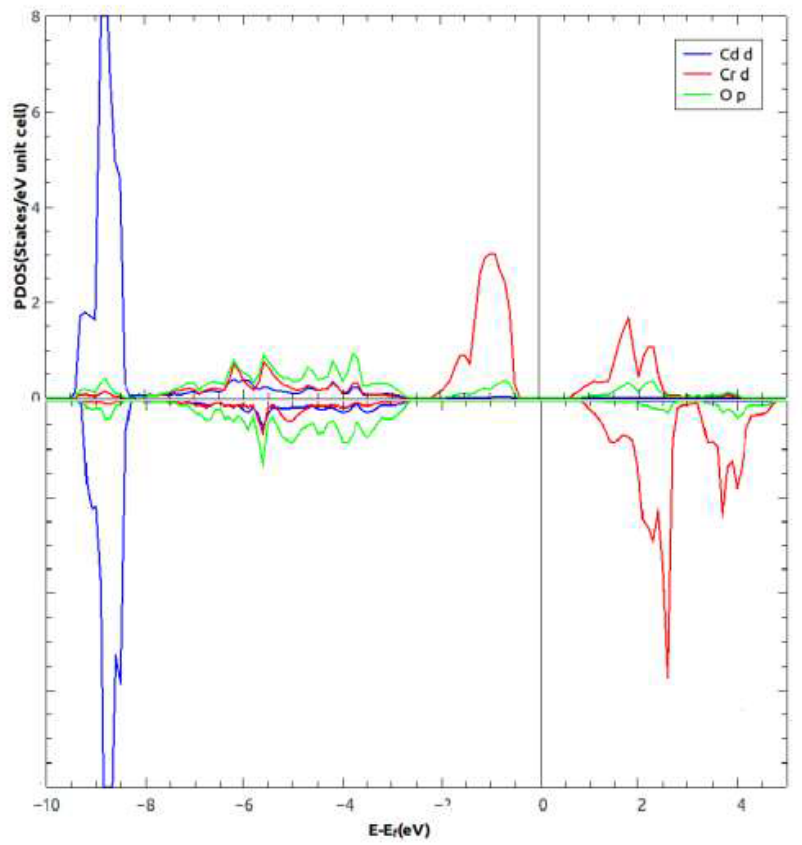

Figure 6: Calculated projected density of states (PDOS) for spinel $\mathrm{CdCr}_{2} \mathrm{O}_{4}$ in ferromagnetic ordering for both spin up and down.

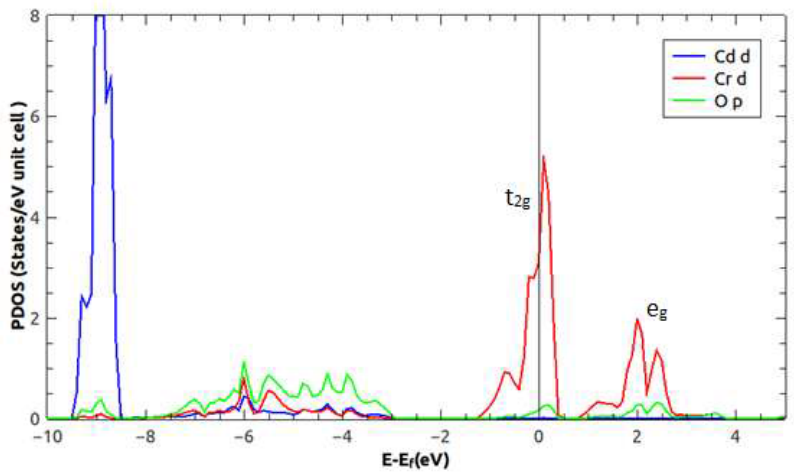

Figure 7: Densities of $\mathrm{Cd} \mathrm{d}$ (blue solid line), $\mathrm{Crd}$ (red solid line), and $\mathrm{O} p$ (green solid line) states in $\mathrm{CdCr}_{2} \mathrm{O}_{4}$. Zero energy is chosen at the Fermi level.

also the structures would have electronic ground state ranging from metallic to semiconductor electronic character in different magnetic orderings.

\section{Acknowledgements}

This work is supported by Universiti Putra Malaysia and Ministry of Higher Education (MOHE) Malaysia, through Fundamental Research Grant Scheme (FRGS) (Project Code: FRGS/1/11/ST/UPM/02/11). Figures showing electronic configurations, charge density and charge density contour are generated using the XCRYSDEN program.

\section{References}

1. Finger LW, Hazen RM, Hofmeister AM (1986) High-pressure crystal-chemistry of spinel $\left(\mathrm{MgAl}_{2} \mathrm{O}_{4}\right)$ and Magnetite $\left(\mathrm{Fe}_{3} \mathrm{O}_{4}\right)$ - Comparisons with silicate spinels. Physics and Chemistry of Minerals 13: 215-220.

2. Yamasaki $Y$ (2006) Magnetic reversal of the ferroelectric polarization in a multiferroic spinel oxide. Physical Review Letters 96: 207204.

3. Hemberger J, Lunkenheimer P, Fishtl R, Loidi A (2005) Relaxor ferroelectricity
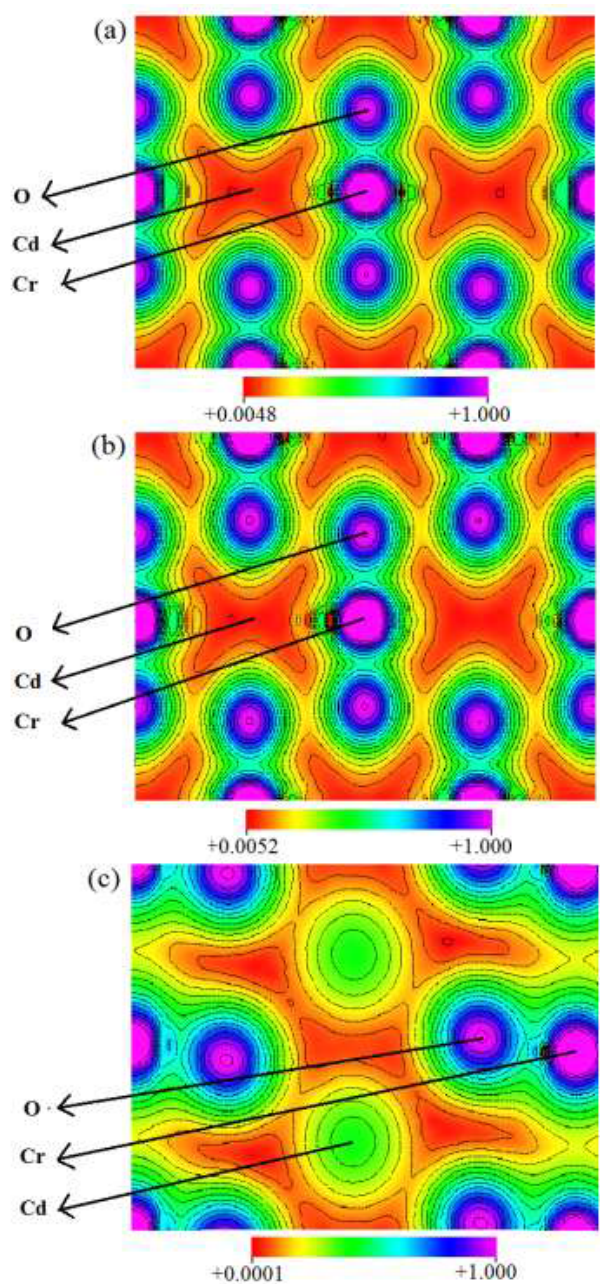

Figure 8: Calculated electronic charge density distribution for spinel $\mathrm{CdCr}_{2} \mathrm{O}_{4}$; (a) Paramagnetic ordering, (b) Ferromagnetic ordering, (c) Antiferromagnetic ordering.

and colossal magnetocapacitive coupling in ferromagnetic $\mathrm{CdCr}_{2} \mathrm{~S}_{4}$. Nature 434: 364-367.

4. Xie X, Li Y, Liu ZQ, Haruta M, Shen W (2009) Low-temperature oxidation of CO catalysed by $\mathrm{Co}_{3} \mathrm{O}_{4}$ nanorods. Nature 458: 746-749.

5. Thackeray MM (1997) Manganese oxides for lithium batteries. Progress in Solid State Chemistry 25: 1-71.

6. Chung JH, Matsuda M, Lee SH, Kakurai K, Ueda H, et al. (2005) Statics and Dynamics of Incommensurate Spin Order in a Geometrically Frustrated Antiferromagnet $\mathrm{CdCr}_{2} \mathrm{O}_{4}$. Physical Review Letters 95: 247204.

7. Lee SH, Louca D, Ueda H, Park S, Sato TJ, et al. (2004) Orbital and Spin Chains in $\mathrm{ZnV}_{2} \mathrm{O}_{4}$. Physical Review Letters 93: 156407.

8. Lee SH, Broholm C, Kim TH, Ratcliff W, Cheong SW (2000) Local Spin Resonance and Spin-Peierls-like Phase Transition in a Geometrically Frustrated Antiferromagnet. Physical Review Letters 84: 3718.

9. Tchernyshyov O, Moessner R, Sondhi SL (2002) Order by distortion and string modes in pyrochlore antiferromagnet. Physical Review Letters 88: 067203.

10. Kumar A, Fennie CJ, Rabe KM (2012) Spin-lattice coupling and phonon dispersion of CdCr2O4 from first principles. Physical Review B 86: 184429.

11. Giannozzi P, Baroni S, Bonini N, Matteo C, Roberto C, et al. (2009) QUANTUM ESPRESSO: a modular and open-source software project for quantum simulations of materials. Journal of Physics: Condensed Matter 21: 395502. 
Citation: Bolandhemat N, Md Mahmudur R, Zainuddin H, Chan KT, Shuaibu A (2017) Density Functional Study of Electronic, Magnetic and Chemical Bonding Properties of Spinel $\mathrm{CdCr}_{2} \mathrm{O}_{4}$. J Material Sci Eng 6: 390. doi: 10.4172/2169-0022.1000390

Page 7 of 7

12. Monkhorst HJ, Pack JD (1976) Special points for Brillouin-zone integrations. Physical Review B 13: 5188.

13. Chung JH, Kang HJ, Ratcli R, Gehring P (2007) The Magnetic Phase Transition and Spin Fluctuations in the Geometrically Frustrated Antiferromagnetic Spinel $\mathrm{CdCr}_{2} \mathrm{O}_{4}$ : An Experiment Using the SPINS Triple-Axis Spectrometer. Summer School on Methods and Applications of Neutron Spectroscopy NIST Center for Neutron Research June 25-29. 